\title{
Bromine Recycling in the Chemical Industry - An Example of Circular Economy
}

\author{
Francis Pilloud ${ }^{* a}$, Nasibeh Pouransaria ${ }^{\text {, Luc Renard }}{ }^{a}$, and Rebecca Steidle ${ }^{b}$
}

\begin{abstract}
This paper discusses the application of the circular economy concept and industrial ecology approach in the context of industrial chemical sites. A real-life case study about the use of bromine as reactant for chemical synthesis and its recycling by Syngenta in Monthey is described in detail. With a recovery yield of $97 \%$ it represents a well-established example of closed loop recycling, one aspect of the circular economy. The process leads to significant safety and environmental risk reduction and economic savings in the order of several million CHF per year.
\end{abstract}

Keywords: Chemical industry $\cdot$ Circular economy $\cdot$ Industrial ecology $\cdot$ Industrial waste management $\cdot$ Material efficiency

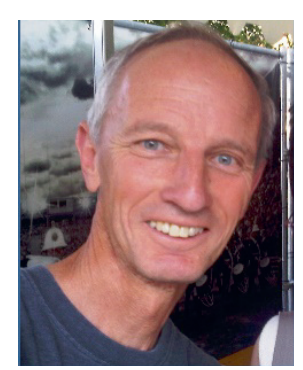

Francis Pilloud holds a PhD in Chemistry from the University of Lausanne. He started his career in the industry in 1988, in Syngenta Crop Protection in Monthey (at the time Ciba-Geigy) in the analytical department. In subsequent years, he successively held many different functions, mainly in production, as a production chemist, process development chemist and production manager, particularly for bromination reactions. After a period as project manager, he took on his current role as Global Environmental Technology Manager at Syngenta.

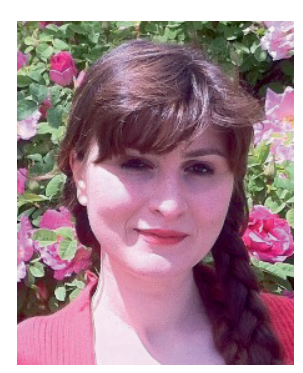

Nasibeh Pouransari PhD graduated from EPFL in energy and mechanical engineering. Her research focused on site-scale process integration and energy efficiency in industry. After a career in public administration on energy efficiency encouragement programs and urban systems, she works since 2016 in Syngenta Crop Protection in Monthey as LEAN process engineer.

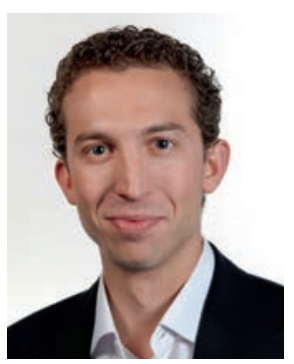

Luc Renard holds a Master in Chemical Engineering from EPFL. He started his career in industry in 2012, in Syngenta Crop Protection in Monthey. After three years in Process Development and Optimization, he moved to HSEQ Department as a Process Risk Assessor. In this role, he performed identification, evaluation and mitigation of risks associated to new and established processes. Since 2016, Luc works in production as an Asset Manager, responsible, among others, for the bromine recovery process. He is also in charge of the design, execution and commissioning of a new bromine recovery installation.

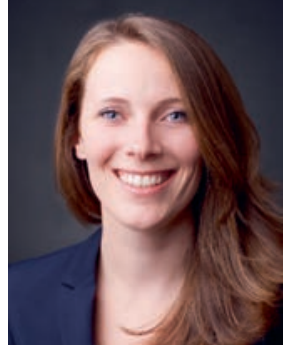

Rebecca Steidle works as an environmental engineer in chemical process development at Syngenta Crop Protection in Münchwilen. In this role she supports the evaluation and thereby consideration of environmental aspects during process development. She holds a Master's degree of environmental engineering from the Technical University of Braunschweig. For her Master's thesis as part of a joint research project with $\mathrm{A} * \mathrm{Star}$ in Singapore she previously worked on methods for sustainability assessment of eco-industrial parks to encourage recycling.

\section{Introduction}

Along with the growing environmental concern and discussions around sustainable economic development, the concept of circular economy (CE) is of great importance. First formally introduced by Pearce and Turner in $1990^{[1]}$ it has since been widely employed, redefined and interpreted in literature. In a frequently cited definition by the Ellen McArthur Foundation, ${ }^{[2]}$ the $\mathrm{CE}$ concept is defined as an economy model which unlike the linear model of economy 'take - make - dispose', aims to preserve the intrinsic value and quality of the products and materials at each step of their use. Given the limited availability of natural resources, the concept of circular economy proposes a model that is restorative and regenerative by design, based on elimination of wastes through the consistent reuse of material. In a circular economy, products are designed for ease of use, disassembly, refurbishment and recycling. The economic growth is mainly based on unlimited resources like labor instead of a linear use of limited natural resources. Rizos et al. ${ }^{[3]}$ classified the CE into three different categories, namely i) using less primary resources, ii) maintaining the highest value of materials and products and iii) changing utilization patterns. They identified eight main CE processes whereby recycling is known as the most traditional one.

Recycling, as the process of reintroducing waste material into new products, is one of the most popular subtopics of CE in literature. ${ }^{[4]}$ Based on the classification by Rizos et al., it is part of 
the 'using less primary resources' category. Recycling includes two main steps: recovery of material and reintroducing the recyclate towards its new usage, therefore it should not be miscomprehended with reuse, which does not include the reprocessing step. While recycling has an economical benefit by saving valuable raw material, it has also several environmental and ecological benefits by reducing the primary resource, energy usage, air and water pollution and by preventing the waste of useful material. Moreover, recycling actively contributes to climate protection and to reduce the greenhouse gas emission. One important factor to have an effective and successful recycling is to achieve the quality required by the new lifecycle of recyclate. Numerous examples of similar resource exchange and internal recycling processes exist and are also discussed in literature. For examples Solanyl ${ }^{\circledR}$, a biopolymer used as a replacement for petroleum-based plastic resins, is cited as an example of the design for closing resource loops due to its bio-based manufacture using potato peels as a recycled and also renewable resource. ${ }^{[5,6]}$ It is biodegradable and compostable according to EN13432 standard. Other examples are the preparation of monoalkyl glyceryl ethers using a side-product from the biobased epichlorohydrin synthesis, ${ }^{[7]}$ and the recycling of end-oflife EPS (expanded polystyrene) containing a brominated flame retardant, consisting of a new dissolving process for the polymer with a final waste stream containing bromine salts that can then be recycled. ${ }^{[8]}$

The concept of circular economy derives from several schools of thought, including the concept of industrial ecology. Industrial ecology focuses on the optimization of energy and material use within industrial systems to reduce waste and maximize material recycling. ${ }^{[9]}$ Industrial clusters provide an ideal setup to experiment with closed material and energy loops. Important synergies can be created through exchange, reuse and recycling of wastes, by-products and energy between neighboring companies, provide a stepping-stone towards creating a circular economy. Having these interactions, the industrial cluster form an industrial symbiosis/ecosystem or an eco-industrial park (EIP) as described by Ehrenfeld and Gertler. ${ }^{[10]}$

In this paper, bromine recycling at the Syngenta production site at Monthey, which is part of an industrial cluster is described to illustrate one aspect of circular economy that is already applied in manufacturing. This recycling process is a closed-loop, integrated in the chemical manufacturing processes of active ingredients for crop protection.

\section{The Global Bromine Market}

Bromine is a starting element for the manufacture of a great number of substances for industry and agriculture. Bromine compounds are used as pesticides, dyestuffs, water purification products and as flame-retardants. Potassium bromide is used as source of bromide ion for the manufacture of silver bromide for photographic film. ${ }^{[11]}$

In nature, bromine is mainly found in the form of bromide salts. It is obtained from seawater, natural brines or from brines generated as a by-product from potash recovery. The concentrations of bromine range from $70 \mathrm{ppm}$ in seawater to $4500-5000$ ppm in the surface layers of the Dead Sea, the richest natural source of bromine on earth. This brine is enriched by solar evaporation until it reaches a concentration of 10-12 g/l. The estimated reserves of bromine in the Dead Sea, expressed in terms of magnesium bromide, are 1000 Mio t. ${ }^{[12]}$ Other bromine sources are located in the USA in natural brine wells in Michigan and Arkansas, in Russia, France and Japan. ${ }^{[13]}$ The world production is around 380 '000 tons per annum (2018), ${ }^{[14]}$ the total production capacity being around 700'000 t/y with about 40\% gained from the Dead Sea.[15]

There is a continuous depletion of natural resources worldwide and also in the Dead Sea the strength of brominated brine is weakening over time. ${ }^{[16]}$ In Asia, bromine availability is very volatile and impacted by seasonal effects (salt fields flooded by monsoon). The trend of the demand is increasing for oilfield mining and fracking as well as for flame retardants that will be used in electric vehicles (due to an inherent risk of potential burning of lithium cells in the batteries). Prices of bromine have been continuously increasing over the last 10 years as a general trend.[16] These trends are strengthening the importance of bromine recycling.

\section{Case Study: Bromine Use and Recycling at Syngenta Monthey}

The chemical industrial site of Monthey in Valais is distinguished with some key aspects of an industrial ecosystem. Four chemicals companies are established in this site, including Syngenta. Services like energy production and waste treatment are externally provided by the company Cimo. These services are commonly used, also logistics are collectively managed by Syngenta for the entire site. Furthermore, $\mathrm{POCl}_{3}$, a by-product from a production process of one of the neighbouring companies is directly used by Syngenta as a reactant, avoiding external supply.

Syngenta has been using bromine in different manufacturing processes for active ingredients on the site of Monthey since the 1980s. As a reactant, bromine is not a chemical element that is part of the final active molecule. Therefore $98 \%$ of bromine ends up in the process waste water in the form of bromide and as organic brominated waste.

The bromine recovery process was integrated within the bromination process since the beginning of its use in Monthey (back to 1980s), allowing for a very low demand of fresh bromine during production.

The introduction of bromine recycling reduces the need for transport and storage which is not recommended due to the dangerous properties of bromine. Bromine is highly reactive with corrosive properties and is toxic: it can cause severe skin burns and eye damage, is fatal if inhaled and very toxic to aquatic life. ${ }^{[17]}$ From an environmental perspective, although the bromide ion has a low toxicity, the emission to water should be minimized.

\subsection{The Bromination Reaction}

Halogenated derivatives are the most common starting reagents for the synthesis of more elaborated structures. Bromine is less reactive than chlorine and so its reaction is slower and more selective. During the multistage chemical synthesis of actives substances, bromine is introduced on a molecule to act as leaving group in a subsequent substitution reaction. This reaction binds one of the bromine atoms to the intermediate and liberates the other, which is captured as hydrobromic acid or as bromide in an aqueous solution. In a second reaction the brominated intermediate is coupled to another building block, which liberates the bromine atom. Finally, the bromine atom is found as bromide ion in the aqueous mother liquor.

The chemical equations for these reactions are written in Eqns (1) and (2).

$A-H \stackrel{B r_{2}}{\longrightarrow} A-B r+H B r$
$A-B r+B \longrightarrow A-B+B r^{-}$

Both reactions are not completed to $100 \%$ and a few percentage of brominated by-products are formed. These impurities are separated in the work-up after the bromination and coupling re- 
action, then usually incinerated as an organic brominated waste. During the work-up, aqueous extraction allows the recovery of bromide salt from the waste water stream.

Due to the high molecular weight of bromine, the bromine need for these reactions is very high in terms of mass. The stoichiometric use gives a consumption of typically 400 to $500 \mathrm{~kg}$ of bromine per ton of final product, assuming a $100 \%$ yield. Given the current volume of production, more than three thousand tons of bromine are processed per year.

\subsection{The Bromine Recovery Process}

\subsubsection{The Bromine Recovery Reaction}

The recovery of bromine from aqueous bromide solution is based on the Kubierschky process, named after its inventor. ${ }^{[18]}$ This process consists of an oxidation of the bromide ion by chlorine, as written in Eqn. (3).

$$
2 \mathrm{Br}^{-}+\mathrm{Cl}_{2} \longrightarrow \mathrm{Br}_{2}+2 \mathrm{Cl}^{-}
$$

\subsubsection{Process Description}

Bromine recovery is a continuous process, consisting of two main steps: the oxidation reaction and a two-step purification process. The flowchart is illustrated in Fig. 1. Due to the corrosive property of bromine, all equipment is made from glass or other corrosive resistant material, such as tantalum-coated steel for the boilers and silicon carbide tube for the heat exchangers.

To allow the reaction, the mixture of aqueous bromide solutions is first acidified to $\mathrm{pH} 1$. Then it feeds the main reactive distillation column (A), after passing through two pre-washing columns (shown in Fig. 2) and a pre-heating exchanger. These columns are used to trap all the vents of the process. Column A is made of glass and is fed by the bromide solution from the top. The gaseous chlorine is injected in the center so that the oxidation reaction takes place on the upper part of the column. At the bottom of the column, steam is introduced to achieve the required reaction temperature of $100{ }^{\circ} \mathrm{C}$ and help strip the bromine vapor which is formed by the reaction and distilled overhead. The water collected at the bottom of the column is drained to the effluent treatment plant (ETP). With 1-2\% of bromide it contains the main loss of the reaction process which has a yield of $98-99 \%$. The bromide concentration in the waste water stream is monitored to control
Fig. 1. The block flow diagram of the bromine recovery process.

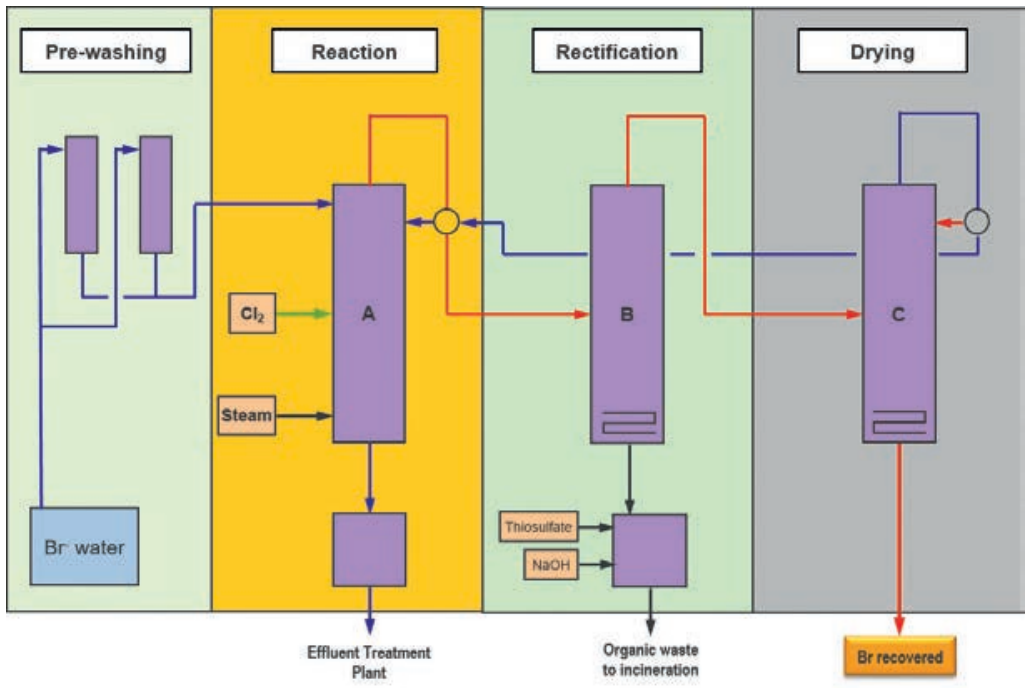

the discharges to the sewer and stop the process in the case of emergency.

The bromine vapor from column $\mathrm{A}$ is then condensed and fed to a second column (B) for purification. The organic impurities collected at the bottom of this column are neutralized with caustic and reduced with thiosulfate. After a solvent extraction step, the organic layer is sent to the incineration unit. This organic waste is only a few tons per year. It contains about $25 \%$ bromine representing $0.05 \%$ of the total bromine involved. The bromine in the flue gas of the incineration process is recovered and recycled as well.

In a third column $(\mathrm{C})$, the bromine stream is separated from traces of water and excess of chlorine through a drying process. The aqueous fraction containing chlorine is decanted and returned to column A. The purified bromine collected at the bottom of this column is then transferred to the storage tank.

\subsection{The Bromine Cycle at Syngenta Monthey}

The bromine cycle on the Monthey site includes several installations, production units and storage tanks that are connected through stainless steel or PTFE-lined pipes. The map presented in Fig. 3 shows the geographical position of these installations and the lifecycle of bromine with the three loops as described below. 
Fig. 3. Geographical representation of the bromine lifecycle with three main loops at Monthey.

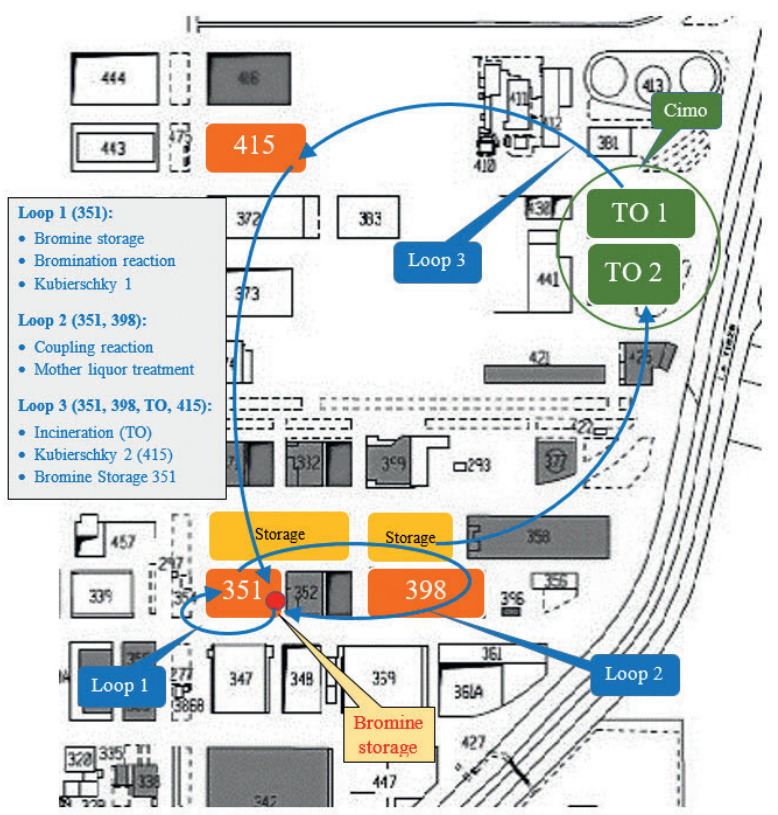

As shown in Figs 3 and 4, the bromine cycle consists of three main loops: From the storage tank the bromine is pumped to the facility for the bromination reaction. The aqueous bromide solution formed in the first step of this reaction is then directly fed to the Kubierschky process and the recovered bromine is sent back
Fig. 4. Mass flow analysis of the bromine recovery process.

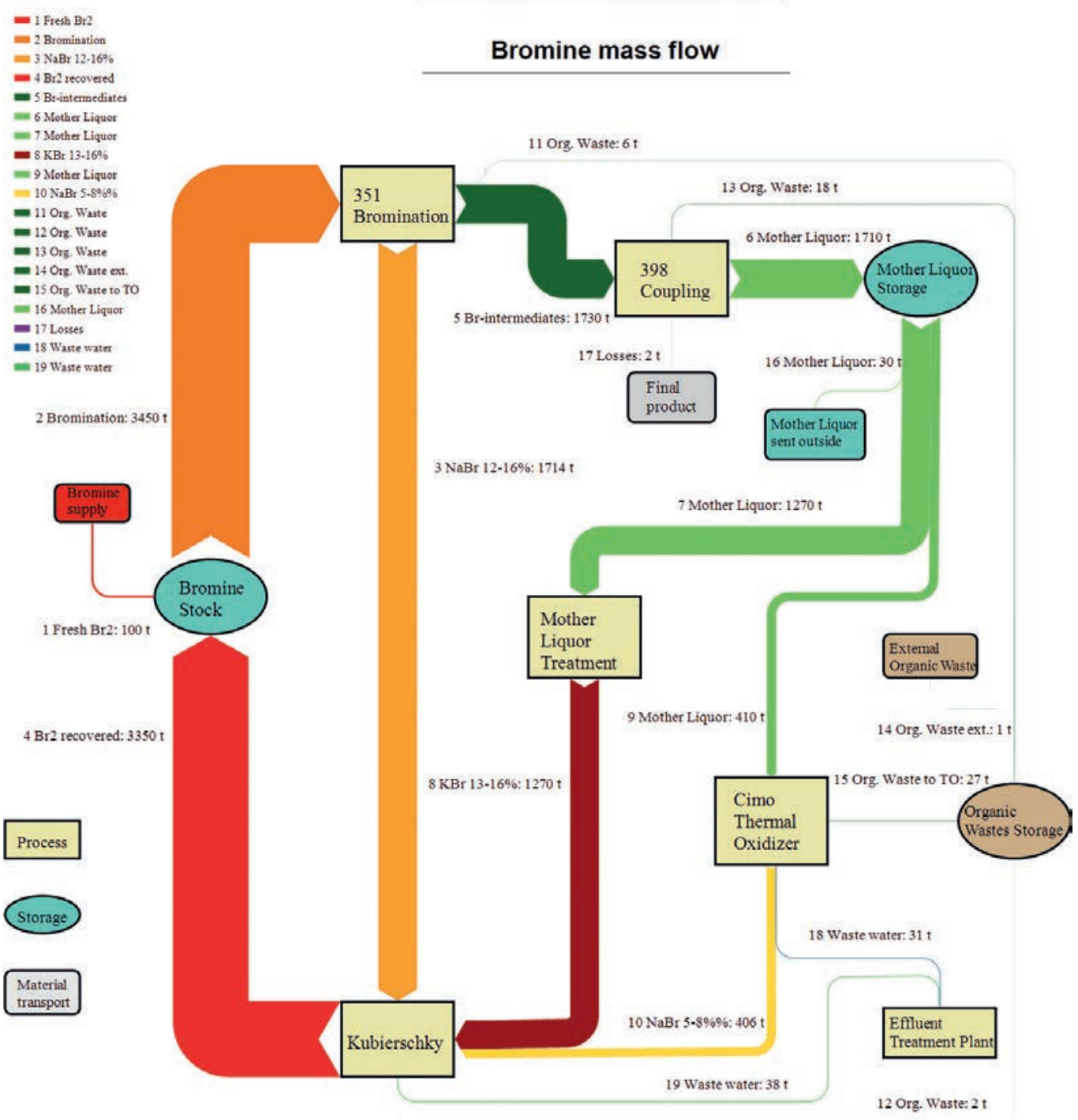


to storage. This is the shortest loop with all assets located in the same production unit (351). It includes $1714 \mathrm{t} / \mathrm{y}$, about half of the total bromine in use. The second loop follows the brominated intermediates which are transferred to the second production unit (398) for the coupling reaction, the second step of the bromination reaction. In the coupling process the bromine is liberated in the form of bromide within the process waste water. Only 2 tons $(0.05 \%)$ of bromine per year are lost from the recycling process in the form of brominated organic impurities contained in the final active ingredient. The mother liquor from this reaction is treated in the same unit (398) by solvent extraction to remove organic impurities, which gives a bromide aqueous solution that is then sent to the Kubierschky installation. The third loop consists of the organic waste that is incinerated at the Cimo Thermal Oxidizer (TO). After incineration, the aqueous bromide solution is transferred to the second Kubierschky installation in unit 415. Due to the capacity limitation of the mother liquor treatment, a portion of this stream is also incinerated.

The Kubierschky installation for bromine recovery is fed with three streams coming from the loops mentioned before:

1. The aqueous solutions of bromide from the bromination steps in unit 351. This stream is a mixture of solutions at different concentration ranging from 4 to $25 \%$ and represents 1714 t of bromine.

2. The aqueous solution from the final active ingredient synthesis in unit 398, containing 13-16\% bromide, which is issued from the mother liquor treatment. This stream represents some 1270 $\mathrm{t}$ of bromine.

3. The bromine recovered from incineration in the Cimo TO represents $406 \mathrm{t}$ bromine. It consists of the organic brominated waste from different chemical processes, containing $27 \mathrm{t}$ of bromine and part of the mother liquor from the coupling process. After incineration, the bromine contained in these waste streams is recovered as aqueous bromide solution from the scrubbers of the TO, which gives the $3^{\text {rd }}$ stream feeding the Kubierschky installation.

Losses of $101 \mathrm{t}(2.9 \%)$ in total occur at three points of the bromine cycle:

1. In the brominated organic impurities contained in the final products: $2 \mathrm{t}(0.05 \%)$.

2. In the waste water from the Kubierschky installation and the TO: $69 \mathrm{t}(2 \%)$.

3. In the mother liquor of the 398 unit which is sent for external treatment: $30 \mathrm{t}(0.87 \%)$.

The losses of bromide from the TO are mainly due to the operating schedule of the incinerator: the wastes containing high concentration of bromine are burned separately during a dedicated operating period, to allow the recovery of bromide from the washing of the flue gas. Wastes with low concentration of bromine are often incinerated together with the other wastes. The bromide content after flue gas washing is therefore too low to allow the recovery.

Bromine from the mother liquor sent for external treatment is also recovered but outside of the cycle described in this article and outside of the control of Syngenta. This external treatment of waste water is regularly not required. However, in the case of capacity issues with the Kubierschky installation, such a treatment is necessary.

The losses must be compensated by purchasing fresh bromine, which amount to $100 \mathrm{t}$ per year $(70 \mathrm{t}$ without external treatment of waste water). A marginal contribution $(1 \mathrm{t})$ comes from the external brominated wastes occasionally incinerated by Cimo.

The material flow analysis of the bromine cycle shows that the total recycling rate of bromine reaches $97.1 \%$, with the new bromine purchase covering only $2.9 \%$ of the overall need of the production process.

The recovery process itself within the Kubierschky installation shows a recovery yield of $98.8 \%$.

\subsection{Economic and Environmental Benefits}

Considering the technical feasibility, the mass balance clearly demonstrates the material efficiency of the bromine recovery cycle.

The cost of recycling bromine, including all processes of the recovery cycle represents about $40-45 \%$ of the current purchase price of bromine, illustrating clearly its economic benefit. The cost of the recycled bromine comes mainly from fixed costs, i.e. asset costs (amortization), energy and labor cost. The variable costs (reactants and chemicals) represent only $20 \%$ of the total cost.

Comparing the current situation with a production without bromine recovery, several million CHF per year are saved. This example illustrates the usage of renewable resources like labor instead of limited natural resources, as suggested in the concept of circular economy.

Considering the quantity of bromine needed for the production of active ingredients at Monthey, the release of this quantity in the environment would have a significant impact on the quality of the river water. It would surely be necessary to find a very costly different way of discharge in order to stay compliant.

Additionally the negative environmental impact of the extraction of bromine from the natural resource and its transport is reduced. The production of fresh bromine from natural resource needs the same Kubierschky process, but as the starting brine is more dilute, the energy consumption is much higher.

From a safety point of view, bromine recycling on site and close to the production units reduces the need for high bromine storage capacity. This represents an important reduction of the risk associated with the storage and transport of large volumes of bromine. The storage of bromine in the form of bromide aqueous solution poses a comparatively much lower risk.

\section{Conclusion and Perspective}

Drawing on the detailed case study of the recycling of bromine in the Monthey industrial cluster, an application of the circular economy concept with its economic and environmental benefits has been presented in this paper.

The recycling of bromine has been implemented in Syngenta Monthey simultaneously with the introduction of bromination reaction to the production process in the 1980s. This is an elaborated example of closed loop recycling with an economic saving of about several million CHF per year.

It has also been shown how an industrial ecology approach can be effectively integrated into an industrial cluster, generating several environmental benefits, by reducing the negative environmental impact of the extraction of fresh bromine from natural resources and the greenhouse gas emission due to lower utility consumption. The production safety is also improved since less bromine storage capacity is required.

During nearly 40 years of bromine use, the production volumes have significantly increased, scaling up the benefits of the recycling process. To improve robustness and allow capacity expansion, both old existing Kubierschky installations will be replaced by a new one in 2019 . Thanks to the price difference between recycled and purchased bromine, the investment can be paid off over a reasonable period, despite a high capital demand. 
[1] D. Pearce, R. K. Turner, 'Economics of natural resources and the environment' Baltimore: Johns Hopkins University Press, 1990.

[2] 'Towards the circular Economy', 'Vol. 1: An economic and business rationale for an accelerated transition', Ellen McArthur Foundation, 2012.

[3] V. Rizos, K. Tuokko, A. Behrens, 'The Circular Economy: A Review of Definitions, Processes and Impacts', Vol. 1, Centre for European Policy Studies: Brussels, Belgium, 2017.

[4] M. Geissdoerfer, P. Savaget, N. M. P. P. Bocken, E. J. Hultink, J. Clean. Prod. 2017, 143, 757.

[5] N. M. P. Bocken, I. de Pauw, C. Bakker, B. van der Grinten, J. Ind. Prod. Engin. 2016, 33, 308

[6] https://www.solanylbiopolymers.com/about-solanyl.html

[7] R. Cucciniello, D. Cespi, Recycling 2018, 3, 22.

[8] Plasteurope.com, in https://www.plasteurope.com/news/RECYCLING_ $t 232726 / 25.11 .2015$.
[9] R. A. Frosch, N. E. Gallopoulos, Sci. Amer. 1989, 198, 152.

[10] J. Ehrenfeld, N. Gertler, J. Ind. Ecol. 1997, 1, 67.

[11] Bromine, Chemicool Periodic Table, Chemicool.com, https://www.chemicool.com/elements/bromine.html 20 Oct. 2012.

[12] 'ICL Global Bromine Industry and its Outlook', http://icl-ip.com/products/ brominel

[13] https://www.lifescience.com/32072-bromine.html

[14] https://www.statista.com/statistics/264926/world-bromine-production/

[15] 'ICL Global Bromine Industry and its Outlook', in http://www.bromine. chem.yamaguchi-u.ac.jp/library/L02_Global\%20Bromine\%20Industry.pdf

[16] S. Lanz, Purchasing Manager, Syngenta Crop Protection, personal communication, 2019.

[17] 'Bromine Safety Date Sheet', Sigma-Aldrich, version 6, 30.03.2016.

[18] K. Kubierschky, United Patent Office no 881 806, 1908. 